\title{
Detektion von Pilz-Melanin mittels einer laser-basierten Fluoreszenzmesstechnik
}

\author{
Natalie Rangno ${ }^{1}$, Katharina Plaschkies ${ }^{1}$, Wolfram Scheiding ${ }^{1}$, \\ Goran Stankovic ${ }^{2}$, Dieter Leupold ${ }^{2}$, Christian Scholz ${ }^{2}$, Matthias Scholz ${ }^{2}$ \\ ${ }^{1}$ Mykolabor Dresden im Institut für Holztechnologie Dresden gemeinnützige GmbH, \\ Zellescher Weg 24, 01217 Dresden, Germany \\ Kontakt: rangno@ihd-dresden.de \\ ${ }^{2}$ LTB Lasertechnik Berlin GmbH \\ Rudower Chaussee 29, 12489 Berlin, Germany
}

\begin{abstract}
Zusammenfassung
Melanine sind natürliche Pigmente der eukaryotischen Zellen, die aufgrund von speziellen physikalischen und chemischen Eigenschaften kompliziert zu diagnostizieren sind. Mittels konventioneller Fluoreszenzuntersuchung (z. B. Anregung der Fluoreszenz mit UV-Strahlung, sog. EinphotonenAnregung) ist Melanin in nativen Pilzen und generell in komplexen organischen Strukturen nicht nachweisbar.
\end{abstract}

Die vorgestellten Untersuchungen befassten sich mit dem Melanin-Nachweis in verschiedenen Pilzen mit einer speziell adaptierten, laser-basierten Fluoreszenzmesstechnik. Das neue LTB-Verfahren zur selektiven Messung der Melaninfluoreszenz basiert auf der lasergestützten Zweiphotonenanregung bei $800 \mathrm{~nm}$ und nutzt den Umstand, dass übliche Fluorophore simultan absorbieren, Melanin hingegen nach schrittweiser Absorption zweier Photonen fluoresziert. Mit dieser neuartigen, unkonventionellen Fluoreszenztechnik gelingt in wenigen Minuten auch die selektive Messung der Melaninfluoreszenz bei verschiedenen Pilzen.

Die Untersuchungen, unter anderem an Trichophyton rubrum, Exophiala dermatitidis und Scopulariopsis brevicaulis, haben gezeigt, dass dieses Verfahren einen empfindlichen und schnellen Nachweis von Melanin in Pilzen ermöglicht.

Schlagwörter: Pilz-Melanin, Melanin-Nachweis, Melaninfluoreszenz, laser-basierte Fluoreszenzmesstechnik.

\section{Einleitung}

Melanine sind natürliche Pigmente und können als multifunktionale Polymere angesehen werden. Sie kommen bei Menschen, Tieren, Pflanzen, Bakterien und Pilzen in verschiedenen Formen vor [1]. Die Melanin-Wirkung in der menschlichen Haut ist ambivalent: positiv als Lichtschutz vor schädlicher UV-Strahlung der Sonne und negativ bei Überproduktion (Hyperpigmentierung).

Bei Überproduktion treten vermehrt dunkle Flecken in der Haut auf (Leberflecken und Sommersprossen), die bösartig zu schwarzem Hautkrebs (Melanom) mutieren können. Melanine spielen aber auch bei Erkrankungen des Menschen eine Rolle, die durch Melaninbildende Pilze verursacht werden (Mykosen).
Melanin wirkt als Pathogenitätsfaktor bei humanpathogenen Pilzen, wie Cryptococcus neoformans oder Candida albicans, infrage, indem es unter anderem an der Beseitigung von für den Pilz schädlichen reaktiven Sauerstoffspezies beteiligt ist. Weiterhin erhöht dieses Pigment bei Pilzen die Resistenz gegen DNAschädigendes UV-Licht [2]. Auf Grund ihrer speziellen physikalischen und chemischen Eigenschaften, wie geringe Löslichkeit, fehlende Kristallisierbarkeit und extrem geringe Fluoreszenz, sind Melanine kompliziert zu diagnostizieren und charakterisieren.

Bis jetzt wurde Melanin makroskopisch durch die bräunliche Verfärbung der Pilzkolonien, mit Hilfe von Melanin-Antikörpern über ELISA (Enzyme-Linked Immunosorbent Assay) nachge- 
wiesen oder aufgrund seiner paramagnetischen Eigenschaften mit Magnetresonanztomographie (MRT) diagnostiziert. Mittels konventioneller Fluoreszenzuntersuchung ist Melanin in nativen Pilzen und generell in komplexen organischen Strukturen nicht nachweisbar. Die Basis der hier vorgestellten Detektion von Pilz-Melanin bildet die speziell adaptierte, laser-basierte Fluoreszenzmesstechnik, von LTB Lasertechnik, Berlin. Diese Detektion-Methode kombiniert den nichtlinear-optischen Effekt einer stufenweisen Zweiphotonen-Absorption mit dessen Induzierung durch einen Pulsdauer-optimierten Farbstofflaser [3].

\section{Material und Methode}

14 Pilzstämme (Hefen, Schimmelpilze und Dermatophyten) wurden auf $4 \%$-SabouraudGlucose-Agar (4 \% SGA) bei $27^{\circ} \mathrm{C}$ für 14 Tage gezüchtet. Drei Nagelproben stammen aus der Routinediagnostik des Labors für medizinische Mikrobiologie, Mölbis. Zur Messung wurde ca. $10 \mathrm{mg}$ Pilzmaterial ohne Substrat bzw. ca. 30 mg Nagelmaterial entnommen und auf einen Glasobjektträger 26 × 76 mm aufgetragen.

Auf der Basis eines neuen patentierten LTB Verfahrens zur Melanomdiagnostik wurde der Nachweis pilzlichen Melanins bei den verschiedenen Pilzen durchgeführt. Das neue Verfahren zur selektiven Messung der Melaninfluoreszenz basiert auf der lasergestützten Zweiphotonenanregung. Zur Anregung des Melanins werden Nanosekunden-Laserimpulse bei $800 \mathrm{~nm}$ benutzt. Diese bewirken im Melanin eine stufenweise Zweiphotonen-Absorption. Alle anderen organischen Komponenten können durch 800 $\mathrm{nm}$ Strahlung nur mittels einer simultanen Zweiphotonen-Absorption zur Fluoreszenz angeregt werden. Somit wird die störende Fluoreszenz aller übrigen Fluorophore weitgehend unterdrückt und die ultraschwache Melaninfluoreszenz wird messbar. Dazu wird ein hochempfindliches Detektionssystem in Verbindung mit einer maßgeschneiderten Laseranregung und Strahlführung benutzt [4]. Dies ist in dem Messsystem LIMES 16-P (LTB Lasertechnik Berlin) realisiert (Bild 1).

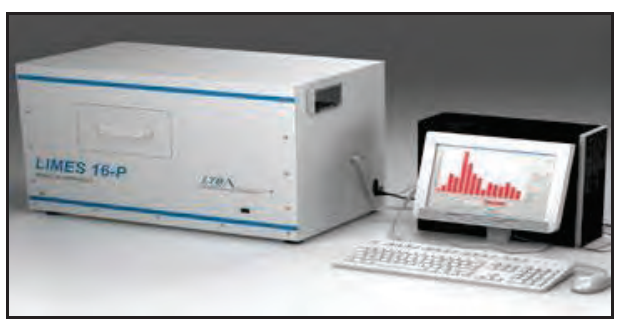

Bild 1 Messsystem LIMES 16-P (LTB Lasertechnik Berlin

\section{Ergebnisse und Diskussion}

Die ersten Ergebnisse zur Detektion von PilzMelanin mittels einer laser-basierten Fluoreszenzmesstechnik sind in Bild 2 dargestellt.

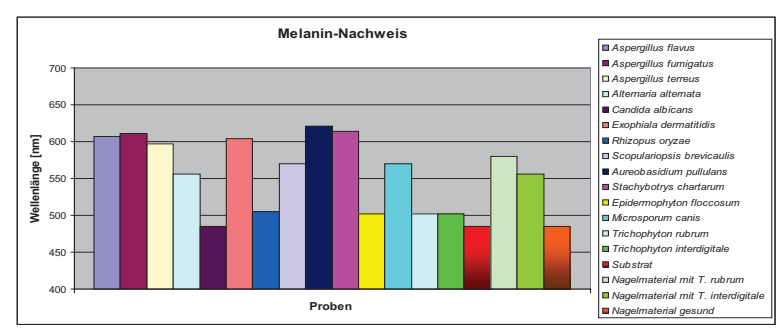

Bild 2 Darstellung der maximalen FluoreszenSpektren des Melanins bei verschiedenen Pilzen und Praxisproben

Wie die ersten Ergebnisse zeigen, unterscheiden sich die Fluoreszenz-Spektren der verschiedenen Pilzspezies voneinander. Die sehr stark melaninhaltigen Pilze wie $A$. pullulans, $E$. dermatitidis und $S$. brevicalius haben maximale Melanin-Spektren ab $600 \mathrm{~nm}$ und schwach melaninhaltige Pilze ab $500 \mathrm{~nm}$. Bei C. albicans konnte nur Autofluoreszenz (485 $\mathrm{nm}$ ) und kein Melanin-Spektrum gemessen werden. Die unterschiedlichen Spektren des Farbpigmentes Melanin sowie deren Intensitäten könnten gattungs- bzw. artspezifisch bei den Pilzen sein. Weiterhin wurde festgestellt, dass bei menschlichem gesunden Nagelmaterial sowie bei dem Substrat (4 \% SGA), sowohl bei der Ein- (Bild 3, dicke Kurve) als auch bei der Zweiphotonenfluoreszenz (Bild 3, dünne Kurve) nur Autofluoreszenz (485 nm) zu sehen ist.

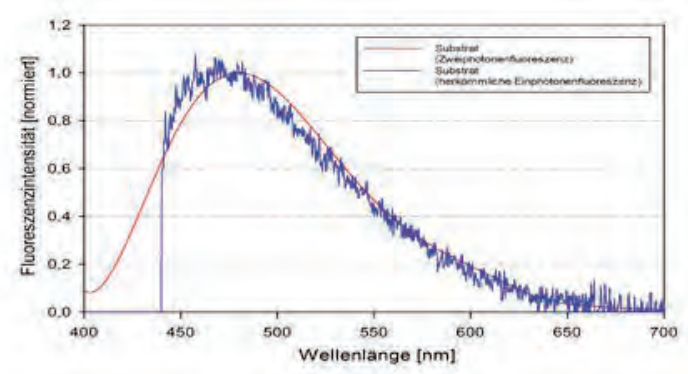

Bild 3 Ein-und Zweiphotonenfluoreszenz-Spektrum des gesundes Nagelmaterials bzw. Substrates $14 \%$ $S G A$ )

Das infizierte Nagelmaterial mit Dermatophyten zeigt nur bei der speziellen Zweiphotonenfluoreszenz eine deutliche Verschiebung des Fluoreszenzmaximums, wie z. B. $580 \mathrm{~nm}$ bei T. rubrum (siehe Bild 3 und Bild 5, dünne Kurve). Die herkömmlichen Einphotonenfluoreszenzspektren zeigen kaum einen Unterschied (Bild 3 bis Bild 5, dicke Kurve). Für die medizinische Pilz-Diagnostik ist diese Aussage interessant. 


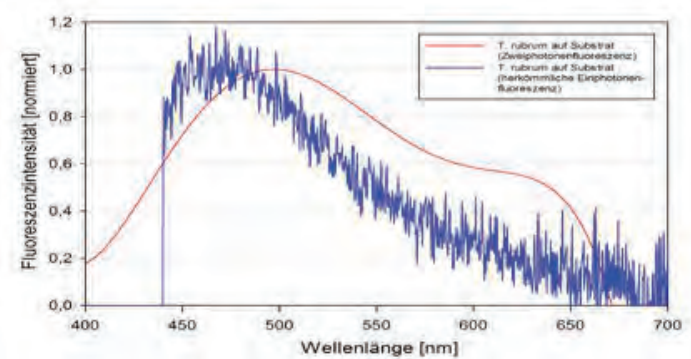

Bild 4 Ein-und Zweiphotonenfluoreszenz-Spektrum von T. rubrum auf Substrat (4\% SGA)

Außerdem zeigte das infizierte Nagelmaterial im Vergleich zur Reinkultur auf Substrat eine deutliche Steigerung des Melaningehaltes (Vergleich Bild 4 und Bild 5).

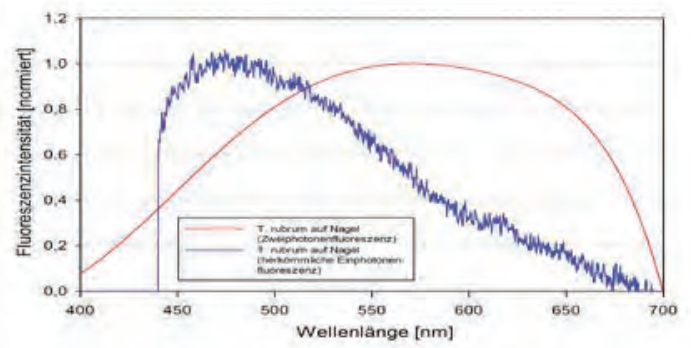

Bild 5 Ein-und Zweiphotonenfluoreszenz-Spektrum des mit T. rubrum infizierten Nagelmaterial

Möglicherweise spielt das Melanin auch bei Dermatophyten als Pathogenitätsfaktor eine Rolle.

\section{Schlussfolgerung}

Die Fluoreszenz von Melanin in der Zelle ist außerordentlich schwach. Liegt das Melanin im Gemisch mit anderen organischen Komponenten (z. B. Pigmente, Aminosäuren und Proteine) vor, so wird die schwache Melaninfluoreszenz von der Fluoreszenz der anderen Komponenten völlig überstrahlt und ist nicht zugänglich. Dies gilt für konventionelle Fluoreszenzmesstechnik (z. B. Anregung der Fluoreszenz mit UVStrahlung, sog. Einphotonen-Anregung). Das neue LTB-Verfahren zur selektiven Messung der Melaninfluoreszenz basiert auf der lasergestützten Zweiphotonenanregung bei $800 \mathrm{~nm}$ und nutzt den Umstand, dass übliche Fluorophore simultan absorbieren, Melanin hingegen nach schrittweiser Absorption zweier Photonen fluoresziert [5]. Mit dieser neuartigen, unkonventionellen Fluoreszenztechnik gelingt in wenigen Minuten auch die selektive Messung der Melaninfluoreszenz bei verschiedenen Pilzen.
Weiterführende Forschungsarbeiten sollen die Relevanz und Nutzbarkeit der Fluoreszenztechnik zum Nachweis und Charakterisierung des pilzlichen Melanins in der medizinischen Diagnostik z. B. zur Früherkennung von Dermatophyten oder Hautmykosen klären. Bei Erfolg könnte direkt am Patienten das verdächtige Haut- bzw. Nagelareal optisch untersucht werden und unnötige Gewebeentnahmen und teilweise lange und psychisch belastende Wartezeiten auf den Befund entfallen

\section{Literatur:}

[1] H. Hof, Mykologie für Mediziner, Thieme, Stuttgart (2008); S 13

[2] R. Morris-Jones, B. Gomez, S. Diez, M. Uran, S. Morris-Jones, A. Casadevall, J. Nosanchuk, A. Hamilton J, Synthesis of Melanin Pigment by Candida albicans In Vitro and during Infection. Infection and Immunity, Vol. 73, No. 9 (2005); S $147-6150$

[3] M. Scholz und D. Leupold, Ortsaufgelöstes Meßverfahren für die Detektion von Melanin in Fluorophorgemischen einer Festkörperprobe, $\mathrm{Pa}$ tentschrift DE 102006029809 (2006)

[4] M. Scholz, G. Stankovic, G. Seewald und D. Leupold, Uncovering of melanin fluorescence in human skin tissue, Proc; SPIE 6633 (2007), 2126

[5] M. Scholz, Früherkennung des schwarzen Hautkrebses erfolgreich getestet, LTB Pressemitteilung (2011) 